\title{
Preliminary examples of 3D vector flow imaging
}

Pihl, Michael Johannes; Stuart, Matthias Bo; Tomov, Borislav Gueorguiev; Hansen, Jens Munk; Rasmussen, Morten Fischer; Jensen, Jørgen Arendt

\section{Published in:}

Proceedings of SPIE

Publication date:

2013

Link back to DTU Orbit

Citation (APA):

Pihl, M. J., Stuart, M. B., Tomov, B. G., Hansen, J. M., Rasmussen, M. F., \& Jensen, J. A. (2013). Preliminary examples of 3D vector flow imaging. In Proceedings of SPIE : Medical Imaging 2013: Ultrasonic Imaging, Tomography, and Therapy (Vol. 8675). SPIE - International Society for Optical Engineering. Proceedings of SPIE - The International Society for Optical Engineering

\section{General rights}

Copyright and moral rights for the publications made accessible in the public portal are retained by the authors and/or other copyright owners and it is a condition of accessing publications that users recognise and abide by the legal requirements associated with these rights.

- Users may download and print one copy of any publication from the public portal for the purpose of private study or research.

- You may not further distribute the material or use it for any profit-making activity or commercial gain

- You may freely distribute the URL identifying the publication in the public portal 


\title{
Preliminary examples of $3 \mathrm{D}$ vector flow imaging
}

\author{
Michael Johannes Pihl ${ }^{a}$, Matthias Bo Stuart ${ }^{a}$, Borislav Gueorguiev Tomov ${ }^{a}$, \\ Jens Munk Hansen ${ }^{a, b}$, Morten Fischer Rasmussen ${ }^{a}$, and Jørgen Arendt Jensen ${ }^{a}$ \\ ${ }^{a}$ Center for Fast Ultrasound Imaging, Dept. of Elec. Eng., \\ Technical University of Denmark, 2800 Lyngby, Denmark. \\ ${ }^{b}$ BK Medical ApS, 2730 Herlev, Denmark
}

\begin{abstract}
This paper presents 3D vector flow images obtained using the 3D Transverse Oscillation (TO) method. The method employs a 2D transducer and estimates the three velocity components simultaneously, which is important for visualizing complex flow patterns. Data are acquired using the experimental ultrasound scanner SARUS on a flow rig system with steady flow. The vessel of the flow-rig is centered at a depth of $30 \mathrm{~mm}$, and the flow has an expected 2D circular-symmetric parabolic profile with a peak velocity of $1 \mathrm{~m} / \mathrm{s}$. Ten frames of 3D vector flow images are acquired in a cross-sectional plane orthogonal to the center axis of the vessel, which coincides with the $y$-axis and the flow direction. Hence, only out-of-plane motion is expected. This motion cannot be measured by typical commercial scanners employing 1D arrays. Each frame consists of 16 flow lines steered from -15 to 15 degrees in steps of 2 degrees in the $Z X$-plane. For the center line, $3200 \mathrm{M}$-mode lines are acquired yielding 100 velocity profiles. At the center of the vessel, the mean and standard deviation of the estimated velocity vectors are $\left(v_{x}, v_{y}, v_{z}\right)=(-0.026,95,1.0) \pm(8.8,6.2,0.84) \mathrm{cm} / \mathrm{s}$ compared to the expected $(0.0,96,0.0) \mathrm{cm} / \mathrm{s}$. Relative to the velocity magnitude this yields standard deviations of $(9.1,6.4,0.88) \%$, respectively. Volumetric flow rates were estimated for all ten frames yielding $57.9 \pm 2.0 \mathrm{~mL} / \mathrm{s}$ in comparison with $56.2 \mathrm{~mL} / \mathrm{s}$ measured by a commercial magnetic flow meter. One frame of the obtained $3 \mathrm{D}$ vector flow data is presented and visualized using three alternative approaches. Practically no in-plane motion $\left(v_{x}\right.$ and $\left.v_{z}\right)$ is measured, whereas the out-of-plane motion $\left(v_{y}\right)$ and the velocity magnitude exhibit the expected $2 \mathrm{D}$ circular-symmetric parabolic shape. It shown that the ultrasound method is suitable for real-time data acquisition as opposed to magnetic resonance imaging (MRI). The results demonstrate that the $3 \mathrm{D}$ TO method is capable of performing $3 \mathrm{D}$ vector flow imaging.
\end{abstract}

Keywords: Medical ultrasound, velocity estimation, three-dimensional vector flow imaging, 3D velocities, volumetric flow rate, transverse oscillation method, spatial quadrature.

\section{INTRODUCTION}

Ultrasonic velocity estimation of the blood is an important diagnostic tool in the clinic. ${ }^{1,2}$ The major breakthrough was the introduction of real-time color flow mapping (CFM) based on the autocorrelation approach. ${ }^{3,4}$ CFM displays the $1 \mathrm{D}$ axial velocities in a $2 \mathrm{D}$ region as only the axial velocity component along the ultrasound beam could be estimated. That has changed with the recent introduction of commercial systems capable of estimating $2 \mathrm{D}$ velocity vectors.

Over the years, several methods have been proposed for vector velocity estimation. The Transverse Oscillation (TO) method ${ }^{5,6}$ is one of these approaches, and it has been implemented on a commercial scanner and FDA approved for clinical use. ${ }^{2,7-10}$

Studies of the hemodynamics in the human circulatory system show that the velocity has components in all three spatial dimensions, and that the blood exhibits complex flow patterns. ${ }^{9,11-13}$ This underlines the need for a method that estimates the instantaneous three-dimensional (3D) velocity vectors. For that purpose, the authors ${ }^{14}$ have suggested the 3D Transverse Oscillation (TO) method. The feasibility of the method has been demonstrated through simulations and experiments. ${ }^{14,15}$

Another modality capable of measuring 3D velocities is Magnetic Resonance Imaging (MRI). ${ }^{11,13,16,17}$ As data acquisition times are long, the data acquisition is typically respiratory compensated and progressively ECG

Further author information: Send correspondence to M. J. Pihl. E-mail: mjp@elektro.dtu.dk 
gated. Consequently, a whole cardiac cycles is reconstructed based on 100-1000 heart beats. Therefore, temporal changes occurring between heart beats cannot be captured by MRI. This severely hampers dynamic studies of heart rate variability as experienced during arrhythmia or as caused by exercise. For the results presented by Kilner et al. ${ }^{11}$ the acquisition of $3 \mathrm{D}$ velocities in a plane took 16 to 24 minutes depending on the heart rate with a time interval of 40-60 ms between successive frames. Ten years later, Markl et al. ${ }^{16}$ described a method for obtaining 3D velocities in a $3 \mathrm{D}$ volume for a time-resolved, reconstructed cardiac cycle. They reported temporal resolutions of 70.6-78.4 ms with data acquisition times of 12-18 minutes. In a review from 2012, Markl et al. ${ }^{17}$ list temporal resolutions ranging from 40 to $80 \mathrm{~ms}$, scan times lasting from 8 to 20 minutes, and spatial resolutions of 0.8-3.0 mm, depending on the anatomic region in question. As data acquisition times are long, the data acquisition is respiratory compensated and, typically, progressively ECG gated. Consequently, a whole cardiac cycles is reconstructed based on several hundred heart beats. Therefore, temporal changes occurring between heart beats cannot be captured by MRI.

The purpose of this paper is to demonstrate the feasibility of employing the 3D TO method for real-time $3 \mathrm{D}$ vector flow imaging (VFI) using ultrasound. For a cross sectional plane of an artifical vessel, preliminary examples of estimated 3D velocities demonstrates the 3D VFI capabilities. Calculations illustrate that real-time data acquisition for 3D VFI in two crossing planes is attainable with a frame rate of $20 \mathrm{~Hz}$.

The following sections present the 3D TO method along with the materials and methods used. In Section 4. The final section concludes on the results and outlines the perspectives.

\section{THE 3D TRANSVERSE OSCILLATION METHOD}

The 3D TO method employs an approach that synthesizes transverse oscillations as suggested by Jensen and Munk ${ }^{5}$ where Anderson ${ }^{18}$ proposed a similar method. The 3D TO method estimates the transverse and elevation velocity components based on two double-oscillating fields and spatial quadrature sampling by employing a $2 \mathrm{D}$ phased array transducer. In the following, the field generation and beamforming as well as the velocity estimation employed in the 3D TO method are described.

\subsection{Field generation and beamforming}

In the 3D case, the concept is to create two double oscillating fields perpendicular to each other. The doubleoscillating fields are synthesized from the same set of received data by applying special apodization functions as illustrated in Fig. 1. To obtain the oscillations in the transverse direction, the receive aperture is modulated in the transverse direction. Conversely, the oscillations in the elevation direction are obtained by modulating the receive aperture in the elevation direction. All TO fields are synthesized from the same data and all components of the velocity vector are obtained simultaneously. The TO fields are created so that they do not oscillate in the perpendicular direction, i.e., the oscillating field in the transverse direction does not oscillate in the elevation direction and vice versa.

Thereby, the velocity estimation is decoupled into three orthogonal and independent velocity components: The axial, $v_{a}$, the transverse, $v_{t}$, and the elevation, $v_{e}$, velocity component. If the direction of the steered beam coincides with the $z$ axis, the three components are equal to $v_{z}, v_{x}$, and $v_{y}$ as illustrated in Fig. 1.

The synthesis of the two double oscillating fields is combined with spatial quadrature sampling. ${ }^{5,18}$ For each discrete depth, two points are sampled in the transverse direction with an inter spacing corresponding to a $90^{\circ}$ phase shift. Similarly, two points are sampled in the elevation direction with a $90^{\circ}$ phase shift in relation to the oscillations in the elevation direction. In addition, samples are obtained along the axis of the steered beam.

For the phased array geometry, the oscillation periods for the transverse, $\lambda_{x}$, and the elevation direction, $\lambda_{y}$, can be estimated $a^{5,19}$

$$
\begin{aligned}
\lambda_{x} & =2 \lambda_{z} \frac{z_{0}}{d_{x} \cos \theta_{z x}} \\
\lambda_{y} & =2 \lambda_{z} \frac{z_{0}}{d_{y} \cos \theta_{z y}},
\end{aligned}
$$



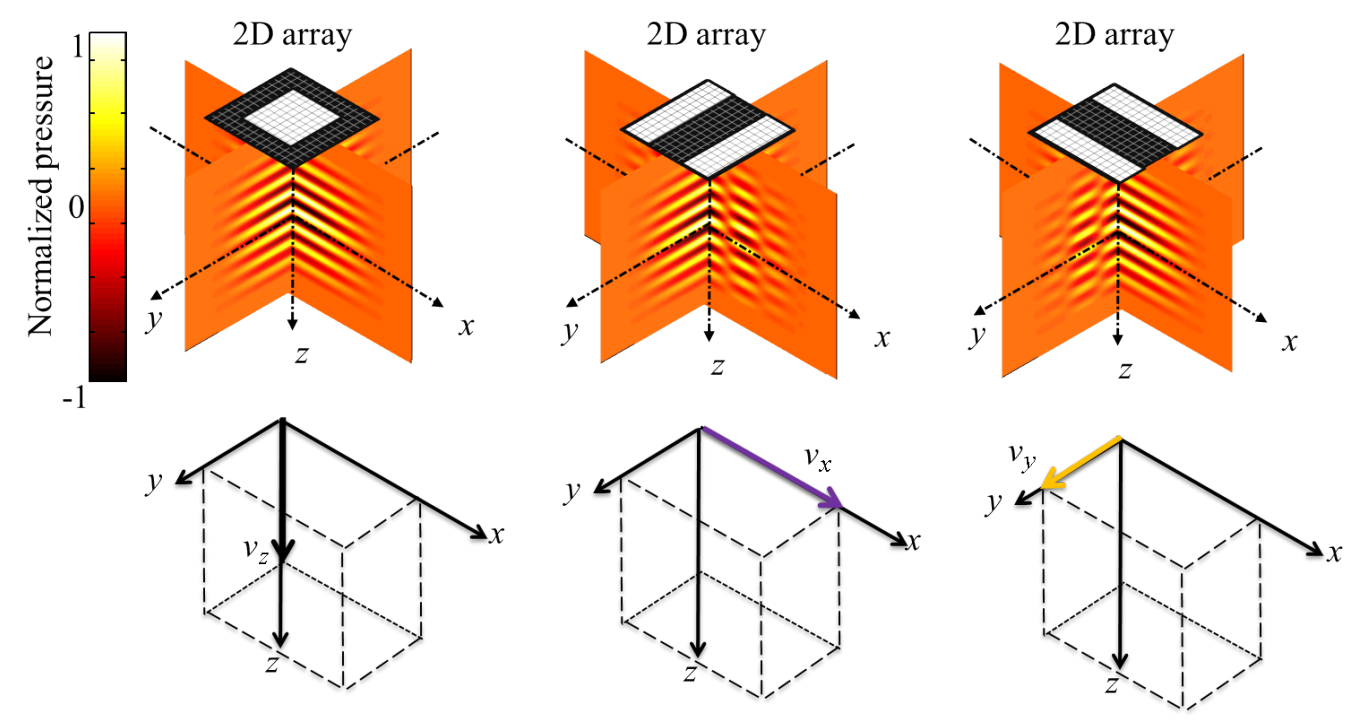

Figure 1. Illustration of the synthesized pulse-echo pressure fields for estimation of the axial, transverse, and elevation velocity component (left, center, and right, respectively). For the axial velocity component there are only oscillations in the axial direction. For the transverse velocity component, the field oscillates in the axial and the transverse direction. For the elevation velocity component, the pressure field oscillates in the axial and the elevation direction. All fields are synthesized in parallel during receive and allows simultaneous estimation of the three velocity components from the same data. The white areas on the $2 \mathrm{D}$ transducers indicate the active elements in receive.

where $\lambda_{z}$ is the axial, temporal wavelength, $z_{0}$ is the radial depth, $d_{x}$ and $d_{y}$ are the distances between the apodization peaks in the $x$ and $y$ direction of the transducer, respectively, and the $\cos \theta_{z x}$ and $\cos \theta_{z y}$ terms account for the steering angle in the transverse $(Z X)$ and elevation $(Z Y)$ plane, respectively.

Improved performance may be obtained, if the inter beam spacing of the two pairs of TO beams is determined by the mean transverse wavelength, $\bar{\lambda}_{x}$, and the mean elevation wavelength, $\bar{\lambda}_{y}$, calculated based on the simulated TO spectra..$^{5,19}$

In total, five lines are beamformed simultaneously in receive from the same data from each emission. For a specific depth, the five samples are: One along the center axis for conventional axial estimation $r_{c}$, and two pairs of the left and right TO samples for spatial IQ in both transverse, $r_{\text {left }_{x}}$ and $r_{\text {right }_{x}}$, and elevation, $r_{\text {left }_{y}}$ and $r_{\text {right }_{y}}$, direction. These samples can be combined to

$$
r_{\mathrm{sq}_{x}}(i)=r_{\text {left }_{x}}(i)+\mathrm{j}_{\mathrm{right}_{x}}(i) \text { and } \quad r_{\mathrm{sq}_{y}}(i)=r_{\text {left }_{y}}(i)+\mathrm{j}_{\mathrm{right}_{y}}(i),
$$

where $i$ is the pulse number of $N_{i}$ emissions. Taking the temporal Hilbert transform or sampling temporal IQ data directly, one obtains

$$
r_{\mathrm{sqh}_{x}}(i)=\mathcal{H}\left\{r_{\mathrm{sq}_{x}}(i)\right\} \quad \text { and } \quad r_{\mathrm{sqh}_{y}}(i)=\mathcal{H}\left\{r_{\mathrm{sq}_{y}}(i)\right\}
$$

The pairwise TO beams are beamformed, so that they are spaced a quarter of the respective oscillation periods apart corresponding to a $90^{\circ}$ phase shift. Thereby, spatial IQ pairs can be obtained in both the transverse and the elevation directions. In the transverse (azimuth) plane, this distance is determined by (1), and in the elevation plane by (2). Because of the limited width of the 2D phased array transducer, lines are beamformed radially. The width also limits the use of an expanding aperture to keep $\lambda_{x}$ and $\lambda_{y}$ constant. Consequently, the transverse and elevation wavelength increase with depth as apparent from (1) and (2).

As a result, the TO lines must be beamformed with an increase in spacing as a function of depth, so that their spacing matches the increasing spatial wavelengths. This can be obtained by beamforming the TO beams 
with a fixed angle, so that they diverge radially. The angle, $\theta_{T O_{z x}}$, between the two TO lines in the transverse direction can be derived as ${ }^{19}$

$$
\theta_{T O_{z x}}=2 \arctan \frac{\lambda_{x} / 8}{z_{0}}=2 \arctan \frac{\lambda_{z}}{4 d_{e}}
$$

where $d_{e}$ is the effective distance between the TO peaks in the apodization function calculated as $d_{e}=d_{x} \cos \theta_{z x}$. Thus, instead of beamforming the TO lines with a fixed lateral distance over depth, they diverge with a fixed angle. When beamforming the other pair of TO lines, $\theta_{T O_{z y}}$ is calculated in the same manner.

The receive apodization for the TO lines will typically be two peaks with a given width, and a given spacing, $d_{x}$ or $d_{y}$. The two pairs of TO lines are beamformed orthogonal to one another, and therefore, the required apodization functions are merely the same except for a rotation of $90^{\circ}$.

All five lines are beamformed in parallel in receive based on the same transmission, so five beamformers in receive are required. The method may be expanded to beamform several flow lines in parallel depending on the calculation capability available. As the lines are beamformed in parallel, the three velocity components are estimated simultaneously.

\subsection{Velocity estimation}

The velocity estimation of the transverse $v_{t}$ and the elevation $v_{e}$ velocity components utilizes the estimator suggested by Jensen. ${ }^{6}$ The axial $v_{a}$ velocity component is calculated using a conventional autocorrelation estimator ${ }^{4}$ with radio frequency (RF) averaging. ${ }^{20}$ The three velocity components are estimated simultaneously from the same data. The velocity estimation is the same for $v_{t}$ and $v_{e}$, each based on one pair of the four TO lines. Based on the four spatial IQ sample pairs from (3) and (4), four new signals can be generated as

$$
\begin{aligned}
& r_{1}(i)=r_{\mathrm{sq}_{\mathrm{x}}}(i)+\mathrm{j} r_{\mathrm{sqh}_{\mathrm{x}}}(i)=\exp \left(j 2 \pi i T_{\mathrm{prf}}\left(f_{x^{\prime}}+f_{p}\right)\right) \\
& r_{2}(i)=r_{\mathrm{sq}_{\mathrm{x}}}(i)-\mathrm{j} r_{\mathrm{sqh}_{\mathrm{x}}}(i)=\exp \left(j 2 \pi i T_{\mathrm{prf}}\left(f_{x^{\prime}}-f_{p}\right)\right) \\
& r_{3}(i)=r_{\mathrm{sq}_{\mathrm{y}}}(i)+\mathrm{j} r_{\mathrm{sqh}_{\mathrm{y}}}(i)=\exp \left(j 2 \pi i T_{\mathrm{prf}}\left(f_{y^{\prime}}+f_{p}\right)\right) \\
& r_{4}(i)=r_{\mathrm{sq}_{\mathrm{y}}}(i)-\mathrm{j} r_{\mathrm{sqh}_{\mathrm{y}}}(i)=\exp \left(j 2 \pi i T_{\mathrm{prf}}\left(f_{y^{\prime}}-f_{p}\right)\right),
\end{aligned}
$$

where the frequency $f_{p}$ is due to the axial pulse modulation, $f_{x^{\prime}}$ is due to the spatial transverse modulation, and $f_{y^{\prime}}$ is due to the spatial elevation modulation. The transverse, $v_{t}$, and the elevation velocity, $v_{e}$, are then calculated by

$$
\begin{aligned}
& v_{t}=\frac{\lambda_{x}}{2 \pi 2 k T_{\text {prf }}} \times \arctan \left(\frac{\Im\left\{R_{1}(k)\right\} \Re\left\{R_{2}(k)\right\}+\Im\left\{R_{2}(k)\right\} \Re\left\{R_{1}(k)\right\}}{\Re\left\{R_{1}(k)\right\} \Re\left\{R_{2}(k)\right\}-\Im\left\{R_{1}(k)\right\} \Im\left\{R_{2}(k)\right\}}\right) \\
& v_{e}=\frac{\lambda_{y}}{2 \pi 2 k T_{\text {prf }}} \times \arctan \left(\frac{\Im\left\{R_{3}(k)\right\} \Re\left\{R_{4}(k)\right\}+\Im\left\{R_{4}(k)\right\} \Re\left\{R_{3}(k)\right\}}{\Re\left\{R_{3}(k)\right\} \Re\left\{R_{4}(k)\right\}-\Im\left\{R_{3}(k)\right\} \Im\left\{R_{4}(k)\right\}}\right),
\end{aligned}
$$

where $T_{\text {prf }}$ is the time between two emissions, $R_{m}(k)$ is the complex lag $k$ autocorrelation value of $r_{m}(k)$ for $m=1, \ldots, 4$. $\Re\{\bullet\}$ and $\Im\{\bullet\}$ denote the real and the imaginary part, respectively. The complex autocorrelation is estimated over $N_{i}$ emissions. RF averaging is performed by averaging the autocorrelation estimate over the length of the excitation pulse. ${ }^{6,20}$

The axial velocity component is calculated using an autocorrelation estimator ${ }^{4}$ with $\mathrm{RF}$ averaging ${ }^{20}$ as

$$
v_{a}=-\frac{\lambda_{z}}{2 \pi 2 k T_{\mathrm{prf}}} \arctan \left(\frac{\Im\left\{R_{c}(k)\right\}}{\Re\left\{R_{c}(k)\right\}}\right),
$$

where $R_{c}(k)$ is the autocorrelation of the center line at lag $k$.

As the beams are steered radially, the estimated velocity components $v_{a}, v_{t}$, and $v_{e}$ must be rotated and scan converted to obtain $v_{x}, v_{y}$ and $v_{z}$. Assuming no steering in the elevation direction, the rotation of the axial, $v_{a}$, and the transverse, $v_{t}$, velocity components to obtain $v_{z}$ and $v_{x}$ is

$$
\left(\begin{array}{c}
v_{z} \\
v_{x}
\end{array}\right)=\left(\begin{array}{cc}
\cos \theta_{z x} & -\sin \theta_{z x} \\
\sin \theta_{z x} & \cos \theta_{z x}
\end{array}\right)\left(\begin{array}{c}
v_{a} \\
v_{t}
\end{array}\right)
$$


A

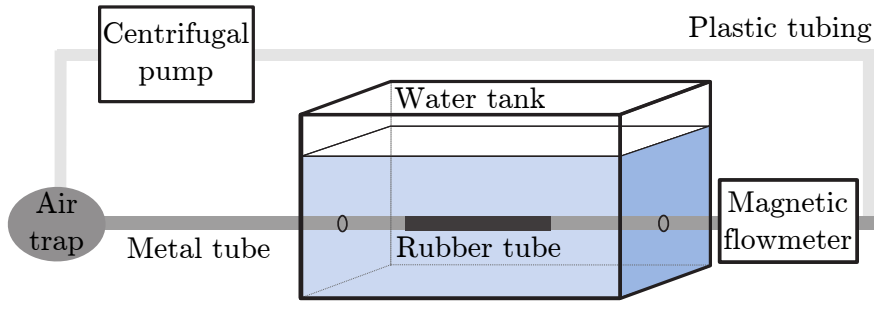

B

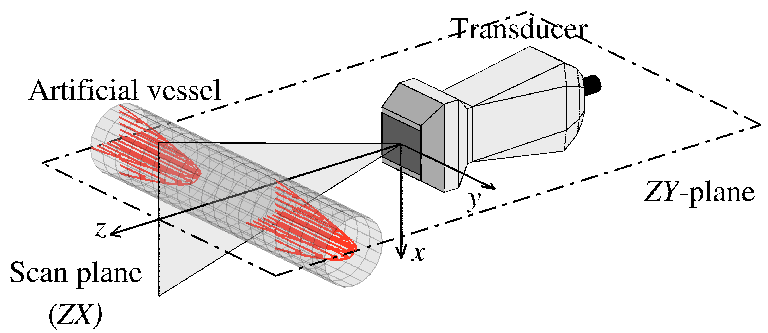

Figure 2. A) Illustration of the flow-rig system. The transducer is placed above the vessel. B) Illustration of the measurement setup. Data are obtained along the $z$-axis (vertically) and in the $Z X$-plane.

where $\theta_{z x}$ is the steering angle in the $Z X$-plane. Due to the position of the scan plane, the elevation velocities, $v_{e}$, are equal to $v_{y}$. Before displaying the $3 \mathrm{D}$ vector flow images, the velocities and the B-mode image are scan converted according to the steering angle of the lines from radial coordinates to Cartesian coordinates using linear interpolation.

\section{MATERIALS AND METHODS}

In the following, the measurement equipment and the data acquisition and processing employed for obtaining the $3 \mathrm{D}$ velocity vectors are described.

\subsection{Measurement equipment}

The 3D Transverse Oscillation method requires a 2D transducer. A $3.5 \mathrm{MHz} 32 \mathrm{x} 32$ element 2D matrix array transducer (Vermont S.A., Tours, France) with a pitch of $0.3 \mathrm{~mm}$ is employed. ${ }^{21}$ At a sampling frequency of $70 \mathrm{MHz}$, data from all the 1024 active elements are acquired simultaneously through the 1024 channels on the synthetic aperture real-time ultrasound system SARUS, ${ }^{22}$ and are stored for offline processing. Velocities are measured in a flow-rig system as illustrated in Fig. 2A. A Cole-Parmer centrifugal pump (Vernon Hills, IL, USA) circulates a blood-mimicking fluid ${ }^{23}$ (Danish Phantom Design, Frederikssund, Denmark) in the closed loop circuit. The vessel radius is $6 \mathrm{~mm}$ and the length of it is long enough to ensure fully developed laminar flow with a parabolic profile. The volume flow rate is measured by a calibrated MAG1100 flowmeter (Danfoss, Nordborg, Denmark) and used for calculating the peak velocity based on the expected parabolic profile. In its concentrated form, the fluid contains $5 \mu \mathrm{m}$-sized orgasol particles dissolved in glycerol, detergent, and demineralized water. For use, it is diluted in a ratio 1:20 with demineralized water, and dextran is added to obtain a viscosity $\mu$ of $3.9 \mathrm{mPa} \cdot \mathrm{s}$. The density $\rho$ is $1.0 \times 10^{3} \mathrm{~kg} / \mathrm{m}^{3}$.

The peak velocity can be calculated based on the flow rate. The flow rate was adjusted to obtain a peak velocity of approximately $1.0 \mathrm{~m} / \mathrm{s}$ to mimic the velocities of the blood in the carotid artery. The pulse repetition frequency was $2.4 \mathrm{kHz}$.

\subsection{Data acquisition and processing}

Ten frames of $3 \mathrm{D}$ vector flow images are acquired in a cross-sectional plane of the vessel orthogonal to the length axis, which coincides with the $y$ axis and the flow direction as illustrated in Fig. 2B. Each frame consists of 16 flow lines steered from $-15^{\circ}$ to $15^{\circ}$ in steps of $2^{\circ}$ in the $Z X$-plane. The angle step corresponds to $\theta_{T O}$ from (3.2), which is this case is $1.7^{\circ}$. The steered beams span a field of view of $30^{\circ}$. At a depth of $30 \mathrm{~mm}$, the transverse extend of the field is $15.6 \mathrm{~mm}$ and enough to cover the vessel.

The velocity estimates are obtained using 32 emissions per estimate. For the center line, the 3200 M-mode lines yield 100 velocity profiles. The scan plane spans the $Z X$-plane, and thereby, the expected flow direction is $\mathbf{v} /|\mathbf{v}|=(0,1,0)$ in $(x, y, z)$. Hence, only out-of-plane motion is expected, which is not measurable by current commercial scanners. 

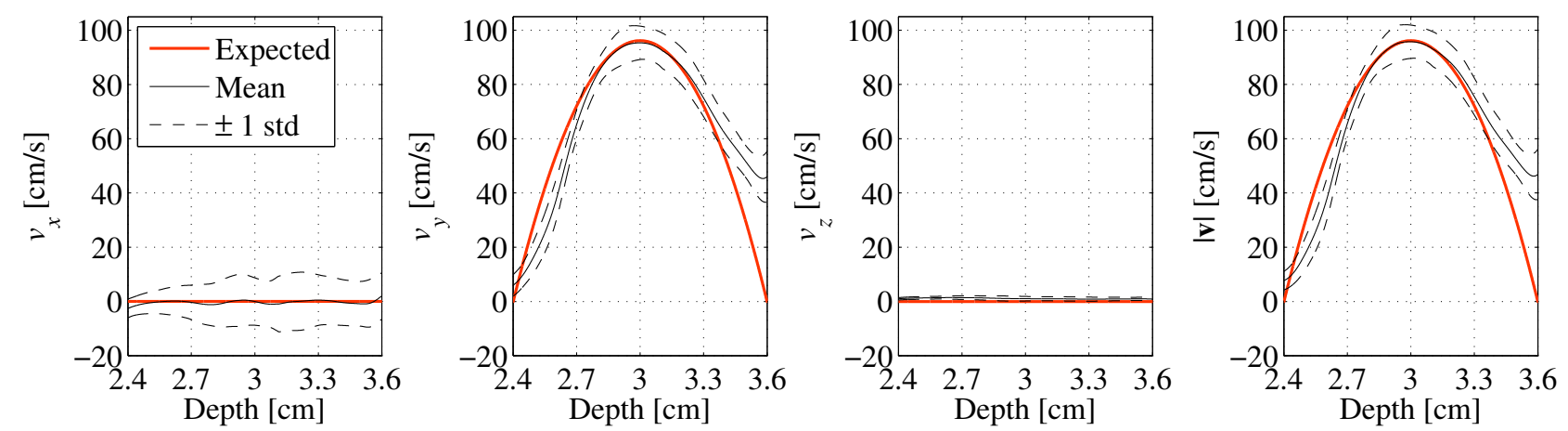

Figure 3. The mean and the range of one standard deviation as well as the expected profiles are plotted for the three velocity components and the resulting velocity magnitude through the vessel along the $z$ axis as illustrated in Fig. 2B.

After matched filtration, the data are beamformed offline using the Beamformation Toolbox $3 .^{24}$ Mean stationary echo cancelling (clutter filtering) is performed by subtracting the mean ensemble value from the 32 M-mode lines prior to the velocity estimation. The discrimination between flow and stationary objects is made by manually outlining the vessel lumen based on the B-mode image at $70 \mathrm{~dB}$ dynamic range.

The volumetric flow rate characterizes the amount of fluid that crosses through a plane per unit time. The flow rate can be calculated by integrating the velocities normal to the scan plane. Using 3D VFI methods, the normal (i.e. out-of-plane) velocity component is obtained. The image scan plane consists of discrete pixels and the associated velocity vectors. In this case, the normal velocity component is $v_{y}$. Hence, the volumetric flow rate $Q_{\text {est }}$ is estimated as

$$
Q_{\mathrm{est}}=p_{A} \sum_{p, q}^{N_{x}, N_{z}} \mathbf{D}(p, q) \mathbf{V}_{\mathbf{y}}(p, q), \quad p=1 \ldots N_{x}, q=1 \ldots N_{z}
$$

where $p_{A}$ is the pixel area, $p$ and $q$ denote the pixels in the axial $z$ and the transverse $x$ direction, respectively, and $N_{x}$ and $N_{z}$ are the number of pixels, respectively. The matrix of out-of-plane velocities is denoted $\mathbf{V}_{\mathbf{y}}$, and $\mathbf{D}$ is the logical discriminator distinguishing between flow (1) or no flow (0).

\section{MEASUREMENT RESULTS AND DISCUSSION}

This section presents and discusses the measurement results including a simple validation example and examples of 3D VFI visualized in three different ways.

\subsection{Validation of the 3D TO method}

The 3200 M-mode lines obtained along the $z$ axis are acquired in order to illustrate the validity of the 3D TO method. Hundred flow profiles are estimated and compared with the expected profile. Fig. 3 shows the velocity profiles for the three velocity components $v_{x}, v_{y}$, and $v_{z}$ and the velocity magnitude obtained along the (top-bottom) diameter of the vessel. The mean of the 100 velocity profiles along with the range of one standard deviation is presented. The mean of the velocities follow the expected profiles. At the center of the vessel, the mean of the measured velocity vector along with the expected velocity and the resulting bias is

$$
\overline{\mathbf{v}}=\left(\begin{array}{c}
\bar{v}_{x} \\
\bar{v}_{y} \\
\bar{v}_{z}
\end{array}\right)=\left(\begin{array}{c}
-0.03 \\
95.3 \\
1.0
\end{array}\right) \pm\left(\begin{array}{l}
8.8 \\
6.2 \\
0.8
\end{array}\right) \mathrm{cm} / \mathrm{s}, \quad \mathbf{v}_{\exp }=\left(\begin{array}{c}
0.0 \\
96.1 \\
0.0
\end{array}\right) \mathrm{cm} / \mathrm{s}, \quad \text { and } \quad \mathbf{B}_{\mathbf{v}}=\left(\begin{array}{c}
-0.03 \\
-0.8 \\
1.0
\end{array}\right) \mathrm{cm} / \mathrm{s} .
$$

The above results and Fig. 3 illustrate the performance of the 3D TO estimator using the given measurement equipment. As the results show, the standard deviation is higher for $v_{x}$ compared to $v_{y}$. This is due to transducer inaccuracies such as phase aberrations and short-circuited elements as well as system noise, which especially affects elements and channels used for estimating $v_{x}$ compared to the ones used for $v_{y}$. Nonetheless, the results demonstrate that the method is capable of estimating $3 \mathrm{D}$ velocities. 


\subsection{Estimation of volumetric flow rate}

During data acquisition the volumetric flow rate is measured using the magnetic flow meter. For the ten frames of $3 \mathrm{D}$ VFI, the measured flow rate was $56.2 \mathrm{~mL} / \mathrm{s}$. From the estimated velocities and using (8), the mean and standard deviation of the 10 frames was $57.9 \pm 2.0 \mathrm{~mL} / \mathrm{s}$. The corresponding bias is $3.0 \%$ relative to the measured value. The obtained volumetric flow rates serve as an additional validation of the 3D TO VFI approach.

Furthermore, the manually outlined cross-sectional area was $112.8 \mathrm{~mm}^{2}$ compared with $113.1 \mathrm{~mm}^{2}$, which is the actual cross-sectional area $\left(\pi r^{2}\right)$ of the vessel. This confirms that the mapped area is of the expected size assuming the scan plane is perfectly orthogonal to the length axis of the vessel.

\subsection{Examples of 3D TO VFI}

Ten frames of $3 \mathrm{D}$ vector flow data in the $2 \mathrm{D}$ scan plane forming a cross section of the vessel are acquired. Due to the flow direction, no in-plane velocity is expected. Hence, conventional 1D or even 2D velocity estimators would not measure any velocity. Visualizing the $3 \mathrm{D}$ velocities acquired in the $2 \mathrm{D}$ plane poses some challenges. Three different approaches to visualize the data is presented below. Note, that for these measurements the expected peak velocity is $99.3 \mathrm{~cm} / \mathrm{s}$.

One of the ten 3D vector flow image frames is visualized in Fig. 4. The figure resembles traditional CFM, as the three velocity components and the resulting velocity magnitude are displayed individually side by side. However, traditional CFM is only able to estimate and visualize the axial velocity. The velocity components $v_{x}$ and $v_{z}$ are almost zero in the scan plane as expected due to the flow direction. For $v_{y}$, the velocity is highest at the center of the vessel and lower at the vessel boundaries. The same appearance is found for the velocity magnitude $|\mathbf{v}|$. Qualitatively, the coloring to some extent has the expected circular-symmetric 2D parabolic velocity profile for $v_{y}$ and $|\mathbf{v}|$.

It should be noted that the black region inside the top of the vessel is an artifact. At the time of acquisition, the strong reflection from the top part of the vessel boundaries saturated the amplifiers due to a too high gain. Consequently, the ringing inside the vessel resulted in signals clipping, and hence, no temporal shifts in the signals are measured during acquisition. This results in an estimated velocity of $0 \mathrm{~m} / \mathrm{s}$.

Visualizing 2D velocities in a plane can be done by various approaches. For example, a circular color wheel can be employed or arrows can represent the direction and the length of the velocity vectors. A combination of the two is also possible. Alternative approaches are streamlines or particle motion visualization. Displaying 3D velocities acquired in a $2 \mathrm{D}$ plane on a screen or paper is somewhat more challenging. In Fig. 5, the out-of-plane velocity component is shown as a $3 \mathrm{D}$ surface plots. Fig. $5 \mathrm{~A}$ shows the velocity profile for a single frame and Fig. 5B the mean velocity profile of the 10 frames. Both the coloring and the height of the surface visualize the velocity component. A disadvantage of this choice of presentation is that the rear side of the surface is not visible. As an aid, iso-velocity contours are viewed below the surface plot.

On the top (left and right), a number of projected velocity slice profiles along with the max velocity projection in both the $x$ and $z$ direction are shown. All in all, the figure illustrates that the velocity profile in the vessel has the expected 2D circular-symmetric parabolic shape. Additionally, the appearance of the mean velocity profile is smoother compared with the single frame as anticipated.

Fig. 5A also shows that the peak velocity for the given frame is $100 \mathrm{~cm} / \mathrm{s}$ as expected. For the mean of the ten frames, the peak velocity is $105 \mathrm{~cm} / \mathrm{s}$, and therefore, slightly higher than the anticipated $99.3 \mathrm{~cm} / \mathrm{s}$. Yet, the expected peak values is within the range of one standard deviation of the measured peak velocity. In case a bias does exist, the velocity estimator may be optimized or calibrated.

A third approach to visualizing the $3 \mathrm{D}$ velocity vectors in the image plan is to plot the $3 \mathrm{D}$ velocity vectors as arrows in 3D space as illustrated in Fig. 6. The arrows indicate the 3D velocity vector with both velocity magnitude and direction. Again, it can be observed that the in-plane velocities are practically $0 \mathrm{~cm} / \mathrm{s}$ as anticipated. For $v_{x}$, the velocity ranges from -2.6 to $2.8 \mathrm{~cm} / \mathrm{s}$, and for $v_{z}$ the range is $-3.3-3.7 \mathrm{~cm} / \mathrm{s}$. This should be compared with $v_{y}$, which range from $0 \mathrm{~cm} / \mathrm{s}$ at the vessel boundary to $102 \mathrm{~cm} / \mathrm{s}$ at the center. Therefore, the velocity vectors are practically parallel with the $y$ direction as expected due to the measurement setup. 
A

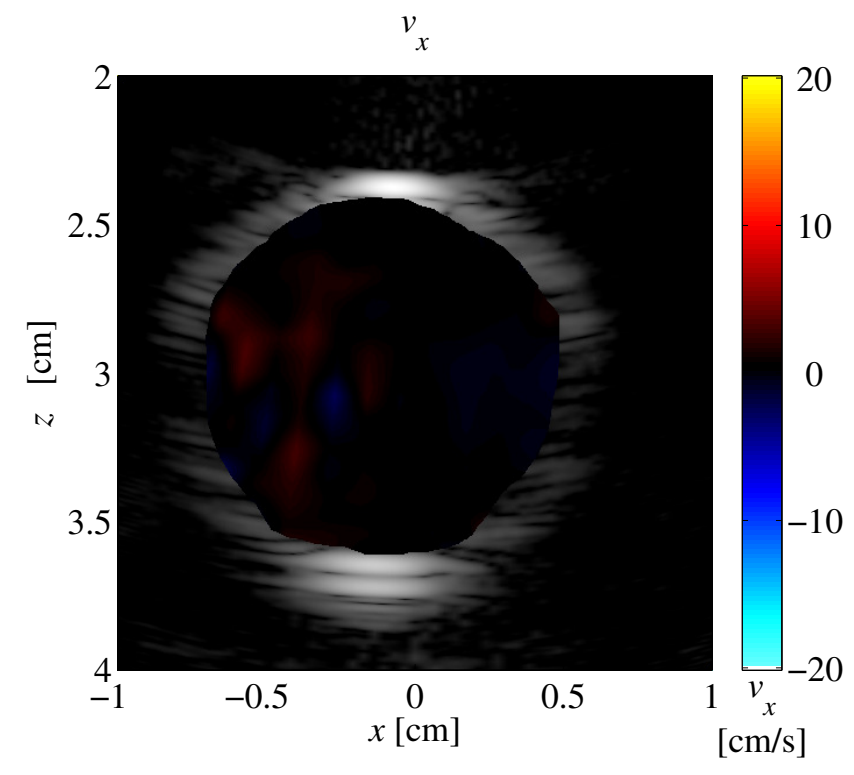

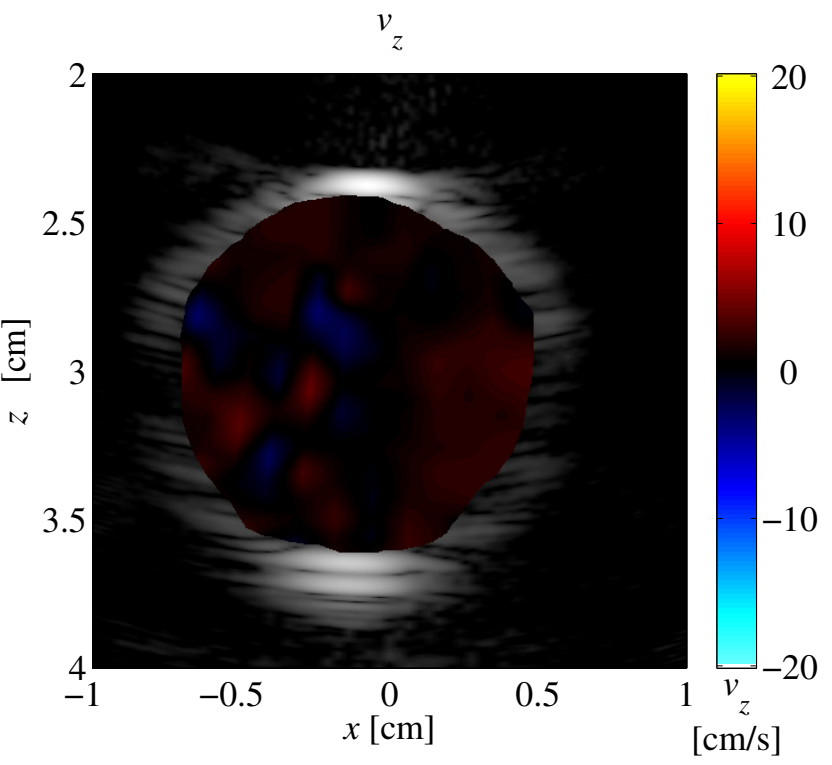

C

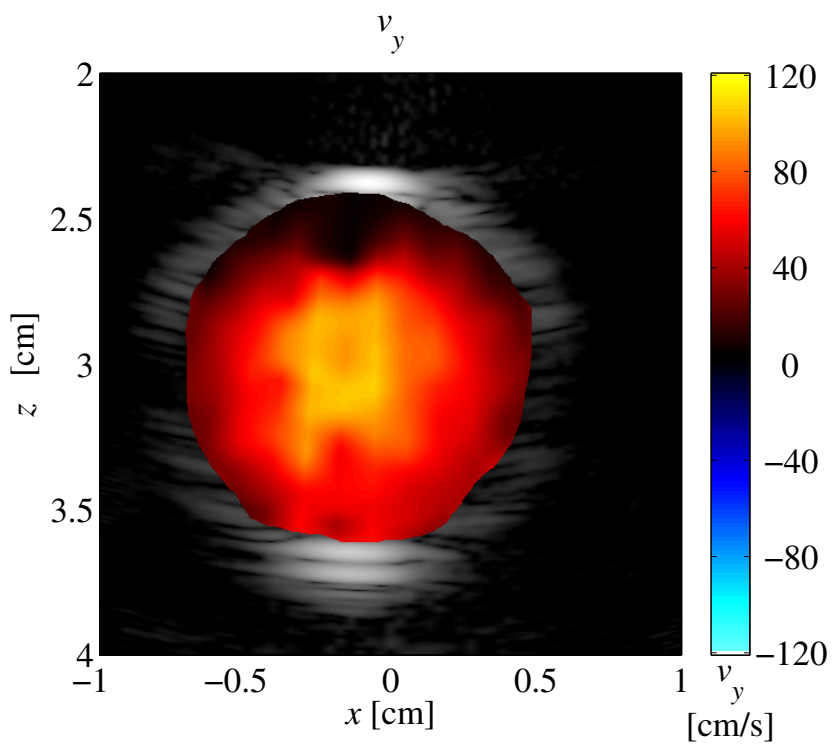

D

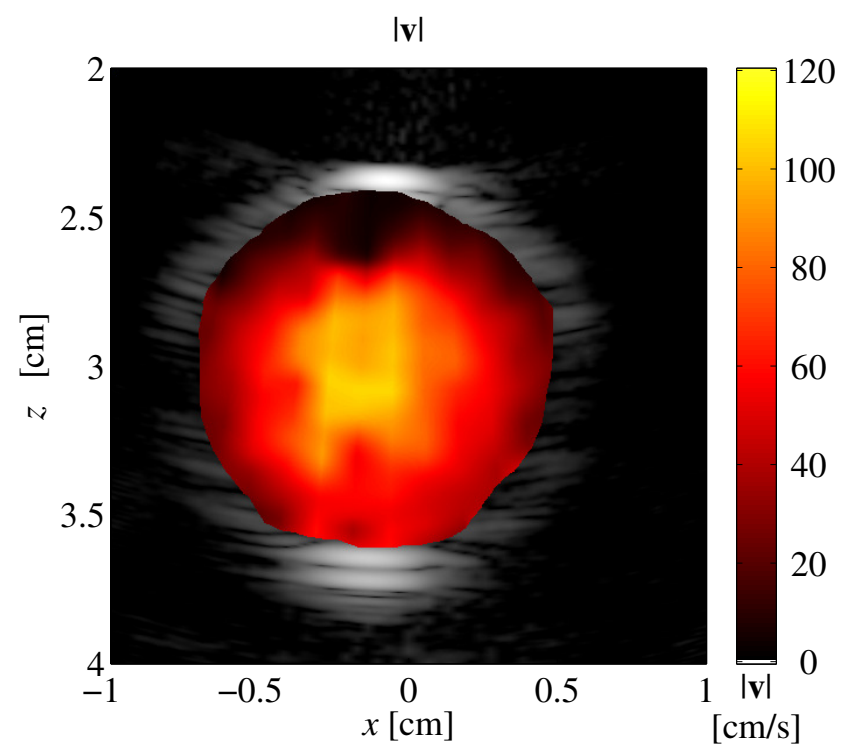

Figure 4. Measured 3D vector flow images in a $2 \mathrm{D}$ scan plane for the three scan converted velocity components $\mathbf{A}) v_{x}$, C) $v_{y}$, and $\mathbf{B}$ ) $v_{z}$, and $\left.\mathbf{D}\right)$ the velocity magnitude. The scan plan is orthogonal to the flow direction. Please note the different scaling of the colorbars. The mask for mapping the flow data was created by manually outlining the vessel lumen based on the B-mode image (70 dB dynamic range). The black area in the top of the vessel lumen is due to clipping in the sampled channel data because of the strong reflections at the top of the vessel. 
A

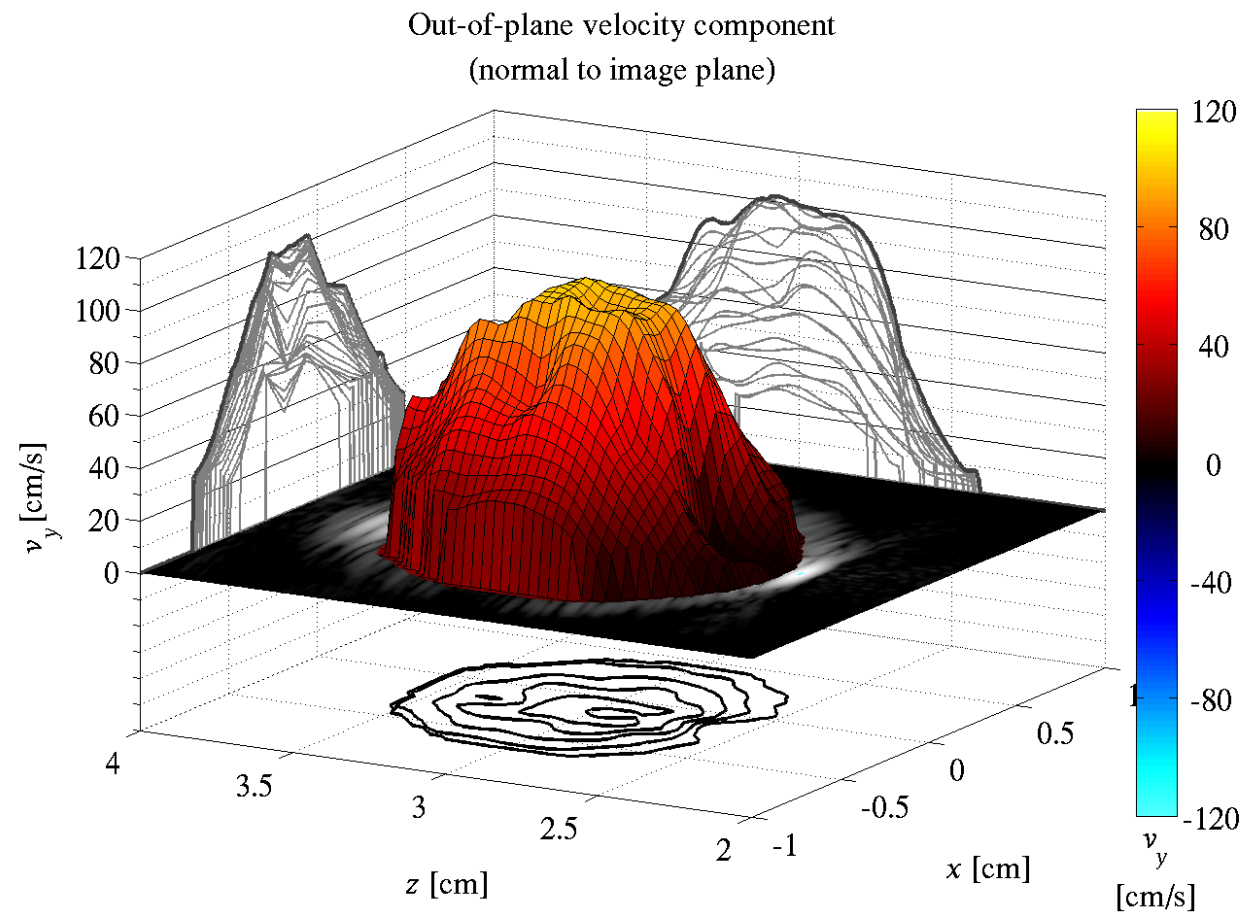

B

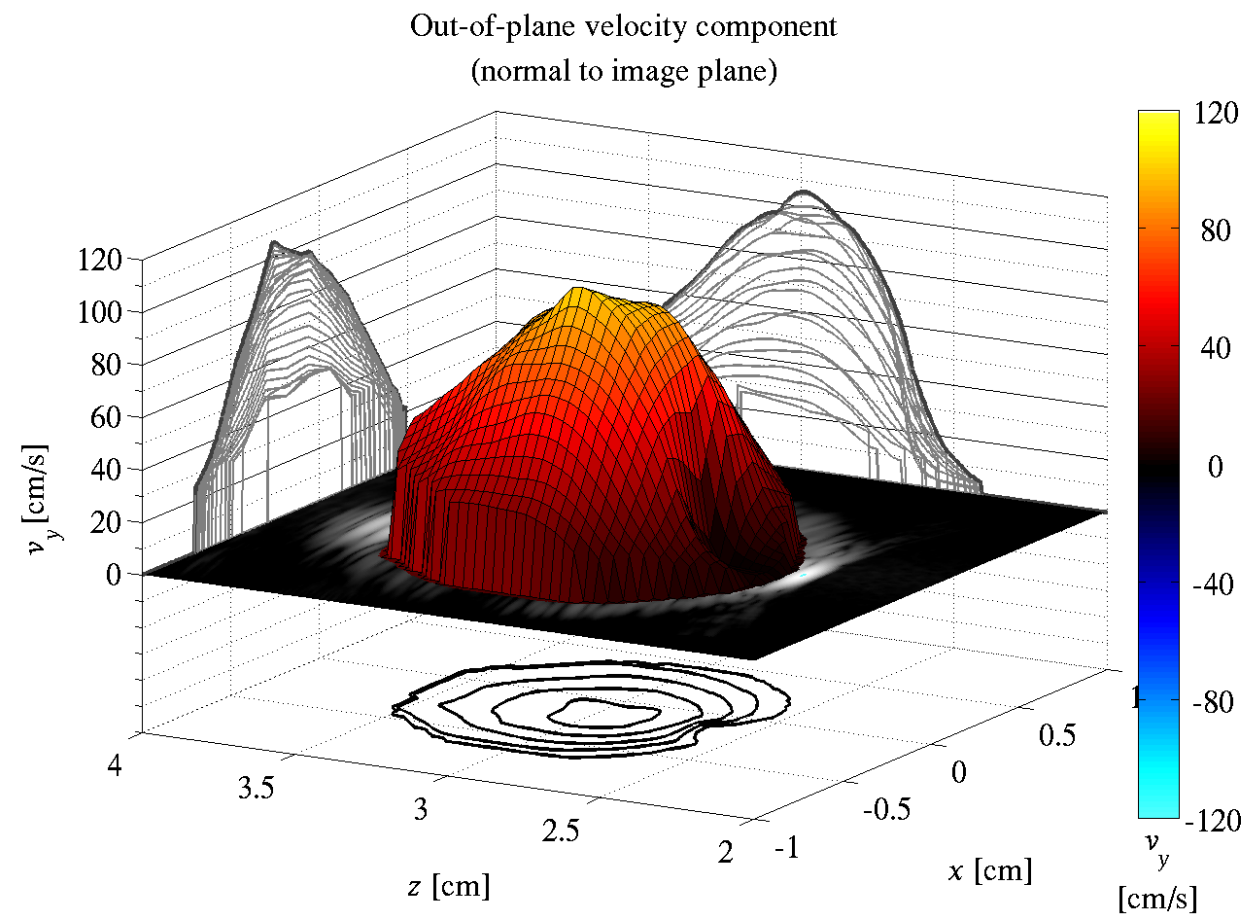

Figure 5. 3D shaded surface plots of the out-of-plane velocity component for A) a single frame and $\mathbf{B}$ ) the mean of 10 frames. The direction of the flow is normal to the B-mode image plane. In this case, the direction coincides with the $y$ direction. The colors indicate the magnitude of the velocity component. The black curves under the plot are iso-velocity contours, the gray curves behind the surface plot are projections of individual slices in the $x$ and $z$ direction, and the thick gray curves on top are the max projections. 


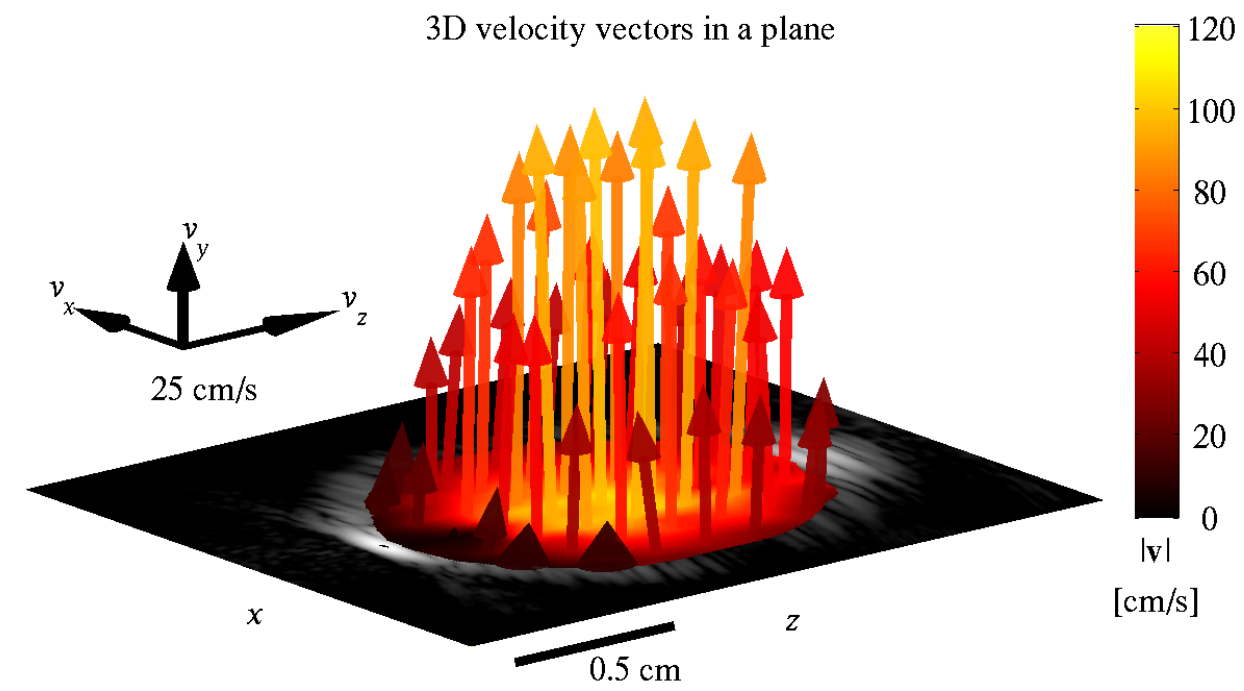

Figure 6. Arrow plot of the $3 \mathrm{D}$ velocities in the $2 \mathrm{D}$ plane for a single frame. The length of the colored arrows as well as the colors indicate the velocity magnitude and the direction of the arrows indicate the direction of the flow. The black bar indicates the dimensions of the B-mode image, and the three black arrows the length of a velocity vector with three equal components of $25 \mathrm{~cm} / \mathrm{s}$.

\subsection{Frame rate considerations compared with MRI}

The results presented in this paper were acquired under steady flow conditions, where frame rate considerations are irrelevant. On the other hand, for pulsatile flow it is important to achieve a sufficiently high frame-rate to capture the temporal changes in the flow and to ensure, that the whole data set is acquire at approximately the same instance in the cardiac cycle.

The number of possible flow lines per frame is

$$
N_{l}=\frac{c}{2 d_{s} f_{r} N_{e}},
$$

where $c$ is the speed of sound in the medium (typically $1540 \mathrm{~m} / \mathrm{s}$ ), $f_{r}$ is the required frame rate, $d_{s}$ is the desired scan depth, and $N_{e}$ is the number of emissions used per velocity estimate.

As stated in Section 1, the temporal resolution is typically $50 \mathrm{~ms}$ for MRI corresponding to a framerate of 20 Hz. Regarding $20 \mathrm{~Hz}$ as a sufficiently high frame rate, scanning down to a depth of $10 \mathrm{~cm}$, and using 16 emissions per estimate, the number of flow lines is approximately 24 . Consequently, a $3 \mathrm{D}$ vector flow image can be created using 24 flow lines. If two cross planes are desired, each plane can consist of 12 flow lines. The field of view may be changed by varying the angle between the flow lines.

These settings are not far from the ones used in the above examples. In fact, if the depth is changed to $4 \mathrm{~cm}$ and $N_{e}$ increased to 32 , a total of 30 flow lines can be acquired per frame. For the results presented above, 16 flow lines were used. Hence, 3D vector flow data can be acquired in two crossing planes with the same field of view as presented here for both plane with a frame rate of about $20 \mathrm{~Hz}$.

Several strategies can be employed to increase the potential number of flow lines: The frame rate may be lowered, the number of emissions used per estimate may be decreased, the scan depth may be reduced, and/or several flow lines may be beamformed in parallel. The options pose various trade-offs between field of view, the variance of the results, and frame rate.

\section{CONCLUSION AND PERSPECTIVES}

Three-dimensional vector flow images using the 3D TO method have been presented, and they demonstrate the feasibility of using the method for 3D vector flow imaging. The mean of 100 profiles along the diameter of the 
vessel demonstrated the performance of the method and validated the method experimentally. Subsequently, 3D VFI was performed in an flow-rig system with steady flow. With the 3D TO method, the full 3D velocity vector including the out-of-plane motion and the correct velocity magnitude can be measured and visualized in various ways. For instance, the measured velocities in the cross section of the vessel exhibits the expected 2D circular-symmetric parabolic profile. Conventional and even 2D methods would fail to estimate any velocity in such a situation. Additionally, the volumetric flow rate through the image plane can be estimated using the method.

The calculations in this paper demonstrate that 3D VFI can be performed in two cross planes with the same temporal resolution as in MRI. However, for MRI the temporal resolution is artificial, as one cardiac cycle is constructed over 100-1000 heart beats. Ultrasound on the other, provides a true temporal resolution in real time. Thereby, temporal changes over the cardiac cycles can be captured at e.g. $20 \mathrm{~Hz}$ and dynamic studies of heart rate variability can be conducted.

With methods for $3 \mathrm{D}$ vector flow imaging, the correct velocity magnitude can be obtained regardless of the orientation of the transducer, and therefore, operator independently. Additionally, the simultaneous calculation of the three velocity components enables visualization of complex flow patterns. Hence, it will be possible to measure and visualize the full 3D vortices and rotational flow found for instance in the carotid artery bifurcation, at stenoses, and at heart and vessel valves.

\section{ACKNOWLEDGMENTS}

The presented work was financially supported by grant 024-2008-3 from the Danish Advanced Technology Foundation and from BK Medical ApS, Denmark.

\section{REFERENCES}

[1] Arning, C., Widder, B., von Reutern, G. M., Stiegler, H., and Görtler, M., "Revison of DEGUM Ultrasound Criteria for Grading Internal Carotid Artery Stenoses and Transfer to NASCET Measurement," Ultraschall in Med 31(3), 251-257 (2010).

[2] Evans, D. H., Jensen, J. A., and Nielsen, M. B., "Ultrasonic colour Doppler imaging," Interface Focus 1, 490-502 (August 2011).

[3] Namekawa, K., Kasai, C., Tsukamoto, M., and Koyano, A., "Realtime bloodflow imaging system utilizing autocorrelation techniques," in [Ultrasound '82], Lerski, R. and Morley, P., eds., 203-208, Pergamon Press, New York (1982).

[4] Kasai, C., Namekawa, K., Koyano, A., and Omoto, R., "Real-Time Two-Dimensional Blood Flow Imaging using an Autocorrelation Technique," IEEE Trans. Son. Ultrason. 32, 458-463 (1985).

[5] Jensen, J. A. and Munk, P., "A New Method for Estimation of Velocity Vectors," IEEE Trans. Ultrason., Ferroelec., Freq. Contr. 45, 837-851 (1998).

[6] Jensen, J. A., "A New Estimator for Vector Velocity Estimation," IEEE Trans. Ultrason., Ferroelec., Freq. Contr. 48(4), 886-894 (2001).

[7] Hansen, P. M., Pedersen, M. M., Hansen, K. L., Nielsen, M. B., and Jensen, J. A., "Demonstration of a vector velocity technique," Ultraschall in Med 32, 213-5 (2011).

[8] Hansen, P. M., Pedersen, M. M., Hansen, K. L., Nielsen, M. B., and Jensen, J. A., "Examples of vector velocity imaging," in [15. Nordic-Baltic Conf. Biomed. Eng. and Med. Phys.], (2011).

[9] Pedersen, M., Pihl, M., Hansen, J. M., Hansen, P. M., Haugaard, P., Nielsen, M., and Jensen, J., "Secondary arterial blood flow patterns visualised with vector flow ultrasound," in [Proc. IEEE Ultrason. Symp.], 1242-1245 (2011).

[10] Jensen, J. A., Pihl, M. J., Olesen, J. B., Hansen, P. M., Hansen, K. L., and Nielsen, M. B., "New developments in vector velocity imaging using the transverse oscillation approach," in [Proc. SPIE Med. Imag.], Accepted (2013).

[11] Kilner, P. J., Yang, G. Z., Mohiaddin, R. H., Firmin, D. N., and Longmore, D. B., "Helical and retrograde secondary flow patterns in the aortic arch studied by three-directional magnetic resonance velocity mapping," Circulation 88(5), 2235-2247 (1993).

[12] Hansen, K. L., Udesen, J., Gran, F., Jensen, J. A., and Nielsen, M. B., "In-vivo examples of complex flow patterns with a fast vector velocity method," Ultraschall in Med 30, 471-476 (2009).

[13] Harloff, A., Albrecht, F., Spreer, J., Stalder, A. F., Bock, J., Frydrychowicz, A., Schollhorn, J., Hetzel, A., Schumacher, M., Hennig, J., and Markl, M., "3D blood flow characteristics in the carotid artery bifurcation assessed by flow-sensitive 4D MRI at 3T," Magn Reson Med 61(1), 65-74 (2009). 
[14] Pihl, M. J. and Jensen, J. A., "3D velocity estimation using a 2D phased array," in [Proc. IEEE Ultrason. Symp.], 430-433 (2011).

[15] Pihl, M. J. and Jensen, J. A., "Measuring 3D velocity vectors using the transverse oscillation method," in [Proc. IEEE Ultrason. Symp.], IEEE (2012).

[16] Markl, M., Chan, F. P., Alley, M. T., Wedding, K. L., Draney, M. T., Elkins, C. J., Parker, D. W., Taylor, R. W. C. A., Herfkens, R. J., and Pelc, N. J., "Time-resolved three-dimensional phase-contrast MRI," J Magn Reson Imaging 17(4), 499-506 (2003).

[17] Markl, M., Frydrychowicz, A., Kozerke, S., Hope, M., and Wieben, O., "4D flow MRI," J Magn Reson Imaging 36(5), $1015-1036$ (2012).

[18] Anderson, M. E., "Multi-dimensional velocity estimation with ultrasound using spatial quadrature," IEEE Trans. Ultrason., Ferroelec., Freq. Contr. 45, 852-861 (1998).

[19] Pihl, M. J., Marcher, J., and Jensen, J. A., "Phased-array vector velocity estimation using transverse oscillations," IEEE Trans. Ultrason., Ferroelec., Freq. Contr. 59, 2662-2675 (December 2012).

[20] Loupas, T., Powers, J. T., and Gill, R. W., "An axial velocity estimator for ultrasound blood flow imaging, based on a full evaluation of the Doppler equation by means of a two-dimensional autocorrelation approach," IEEE Trans. Ultrason., Ferroelec., Freq. Contr. 42, 672-688 (1995).

[21] Ratsimandresy, L., Mauchamp, P., Dinet, D., Felix, N., and Dufait, R., "A 3 MHz two dimensional array based on piezocomposite for medical imaging," in [Proc. IEEE Ultrason. Symp.], 1265-1268 (2002).

[22] Jensen, J. A., Holten-Lund, H., Nielson, R. T., Tomov, B. G., Stuart, M. B., Nikolov, S. I., Hansen, M., and Larsen, U. D., "Performance of SARUS: A Synthetic Aperture Real-time Ultrasound System," in [Proc. IEEE Ultrason. Symp.], 305-309 (Oct. 2010).

[23] Ramnarine, K. V., Nassiri, D. K., Hoskins, P. R., and Lubbers, J., "Validation of a new blood mimicking fluid for use in Doppler flow test objects," Ultrasound Med. Biol. 24, 451-459 (1998).

[24] Hansen, J. M., Hemmsen, M. C., and Jensen, J. A., "An object-oriented multi-threaded software beamformation toolbox," in [Proc. SPIE Med. Imag.], 7968, 79680Y 1-9 (March 2011). 